\title{
Histone Acetyltransferases as Regulators of Nonhistone Proteins: The Role of Interferon Regulatory Factor Acetylation on Gene Transcription
}

\author{
Atsuko Masumi \\ Department of Safety Research on Blood and Biological Products, National Institute of Infectious Diseases, Gakuen, \\ 4-7-1 Musashimurayama-shi, Tokyo 208-0011, Japan \\ Correspondence should be addressed to Atsuko Masumi, amasumi@nih.go.jp \\ Received 12 August 2010; Accepted 5 December 2010 \\ Academic Editor: Patrick Matthias \\ Copyright () 2011 Atsuko Masumi. This is an open access article distributed under the Creative Commons Attribution License, \\ which permits unrestricted use, distribution, and reproduction in any medium, provided the original work is properly cited. \\ When studying transcription factors, it is necessary to investigate posttranslational modifications. Histone acetyltransferases \\ (HATs) are typical of the modification enzymes involved in chromatin regulation. HATs acetylate the transcription factors \\ (nonhistone proteins) as well as histones. Interferon regulatory factors (IRFs) are transcription factors that bind to the interferon \\ regulatory element (IRF-E) and are involved in regulating cell growth, differentiation, and the immune and hematopoietic systems. \\ During the process of binding to a specific DNA element, IRFs also bind to coactivators such as HATs and become modified. This \\ review looks at how IRFs associate with HATs, p300, and PCAF, and thereby contribute to transcriptional activation.
}

\section{Introduction}

Cellular proteins are posttranslationally modified by various mechanisms, including acetylation, deacetylation, phosphorylation, and methylation. The reversible acetylation of histone and nonhistone proteins plays a key role in maintaining cellular homeostasis $[1,2]$. The acetylation of histones is a prerequisite for transcriptional activation. Transcription factors are recruited to the chromatin by acetylated histones, thereby leading to transcriptional activation. In addition to transcription, the status of histone acetylation may influence cell growth and differentiation [3]. Similarly, the acetylation of several transcription factors may regulate their function, by multiple mechanisms, including the modification of DNA binding ability, secondary protein-protein interactions, protein half-life, and protein localization. These events, in turn, can influence DNA repair, cell cycle progression, apoptosis, and various signaling pathways. Many different transcription factors that are regulated through acetylation have been reported over the past decade.

Interferon regulatory factors (IRFs) were characterized as interferon- (IFN-)responsive transcription factors and were investigated through immune response to pathogens, immunomodulation, and hematopoietic development $[4,5]$. As we will describe below, IRFs are transcription factors that have been shown to be acetylated by histone acetyltransferases (HATs) [6, 7]. Such IRFs modifications are associated with cell growth and differentiation as well as the IFN response. IRFs also associate with several HATs to regulate the transcription of specific genes. Here, we introduce a role for acetylated IRFs or IRF-HAT complexes in specific signaling pathways and cell functions.

\section{Histone Acetyltransferases (HATs)}

There is a growing body of evidence supporting the notion that acetylation, like phosphorylation, is an important regulatory protein modification. HATs are increasingly being recognized as modifiers of both histones and nonhistone proteins [2]. Many HATs have been identified such as the GNAT superfamily (PCAF, GCN5), p300/CBP, MYST family proteins including MOZ, and nuclear receptor coactivators. HATs function enzymatically by transferring an acetyl group from acetyl-coenzyme A (acetyl-CoA) to an $\varepsilon$-amino group of certain lysine side chains within a histone's basic $\mathrm{N}$-terminal tail region. Lysine acetylation neutralizes 
part of a histone tail region's positive charge, resulting in weakened histone-DNA or nucleosome-nucleosome interactions [8]. Acetylated chromatin prefers to associate with transcription factors. HATs also acetylate lysine residues within transcription-related proteins as well as histones. HAT proteins form multiple complexes and are recruited to chromatin to acetylate histones or to transcription factors. In terms of IRF regulation by acetylation, PCAF and CBP/p300 play especially important roles.

\section{The IRF Family of Transcription Factors}

Interferon regulatory factors (IRFs) are transcription factors which participate in the early host responses to pathogens, immunomodulation, and hematopoiesis $[5,9]$. Nine mammalian members of the IRF family (IRF-1, -2, -3, -4, -5, $-6,-7,-8$, and -9 ) have been identified. All IRFs carry a conserved DNA-binding domain (DBD) consisting of $\sim 110$ amino acids in the $\mathrm{N}$-terminal region. The $\mathrm{DBD}$ contains a unique helix-turn-helix motif, and it is responsible for binding to the IFN-regulatory factor element (IRF-E) present in the IFN- $\beta$ promoter $[10,11]$. Notably, the consensus sequence of the promoter, G(A)AAAG/CT/CGAAAG/CT/C, is almost indistinguishable from the interferon-stimulated response element (ISRE) activated by IFN signaling. IRF function directly correlates with its sequence-specific binding to the promoters of its regulatory genes. Various posttranslational modifications of IRF proteins affect a host's response to pathogens as well as hematopoietic differentiation, immunomodulation, and oncogenesis.

Among the members of the IRF family, IRF-1 and IRF2 were originally identified through transcriptional studies of the human IFN- $\beta$ gene $[10,11]$. Both IRF- 1 and IRF2 expressed in most cell types and are type I IFN and type II IFN inducible protein. Although IRF-1 and IRF2 have a high ability of binding to IRF-E, they have opposing activities. Whereas IRF-1 activates transcription from promoters carrying the ISRE, IRF-2 represses the transcription of these promoters in IFN system. Both IRF1 and IRF-2 mRNAs are expressed at low constitutive levels in the cell, but the IRF-2 protein is more stable and thus accumulates at higher levels (the half-lives of IRF-1 and IRF-2 are $30 \mathrm{~min}$ versus $8 \mathrm{~h}$, resp.) [12]. In addition, IRF-1 also acts as a tumor suppressor, and IRF-2 induces cellular transformation [12].

\section{Interaction of Histone Acetyltransferases (HATs) with IRF-1 and IRF-2}

We previously demonstrated that IRF-1 and IRF-2 both interact with the histone acetyltransferase PCAF in vitro and that this interaction plays an important role in controlling transcription from relevant promoters $[6,13,14]$. IRF1 stimulates IFN- $\beta$ promoter with PCAF whereas PCAF enhances IRF-2-dependent $\mathrm{H} 4$ promoter activation. Thus, PCAF binding to the IRFs enhances the IRFs-activated promoter. We also demonstrated that the IRF-2 DNAbinding domain interacts with the PCAF bromodomain
[13]. Bromodomain is identified by sequence alignment as a $\sim 60$-amino acid motif conserved among Drosophila Brahma and female-sterile homeotic (fsh) genes and four other potential transcription regulators [15]. Among HATs, PCAF, GCN5, p300, and CBP are bromodomain-containing proteins. The bromodomain of PCAF has a specific affinity for acetyllysine-containing motifs [16]. PCAF bromodomain may associate with the acetylated lysine residue (Lys-75 and Lys-78) within the IRF-2 DNA-binding domain [14]. Although both IRF-1 and IRF-2 are acetylated in vitro [6], the level of acetylation is significantly higher for IRF-2 than for IRF-1. IRF-2 is described as a transcriptional repressor of IFN-responsive genes, and it appears to function by competing with the transcriptional activator IRF-1. We have demonstrated that acetylated IRF-2 inhibits p300-mediated acetylation of core histones [6]. Phorbol esters such as 12O-tetradecanoylphorbol-13-acetate (TPA) activate protein kinase $\mathrm{C}$ and stimulate the differentiation of U937 cells toward macrophage-like cells. Under these conditions, TPA treatment induces the acetylation of nuclear core histones. IRF-2 associates with p300 and PCAF and becomes acetylated in TPA-treated U937 cells. Acetylated IRF-2 reduces the acetylation of histones $\mathrm{H} 2 \mathrm{~A}$ and $\mathrm{H} 2 \mathrm{~B}$ in TPA-stimulated U937 cells. One interpretation of this observed inhibition is that IRF-2, by acting as a substrate for histone acetylases, competitively inhibits histone acetylation (Figure 1(a)). IRF2 inhibition of histone acetylation is relevant to IRF-2's transcriptional repression of IFN-responsive genes. Another IRF member, v-IRF (derived from Kaposi's sarcoma-associated herpes virus) interacts with p300 to similarly inhibit core histone acetylation, resulting in the repressed transcription of interferon-responsive genes [17].

In contrast, IRF-2 can act as a positive regulator for ISRE-like sequences such as the H4 promoter [18, 19]. IRF2 is acetylated during the active growth of NIH3T3 cells (i.e., nonconfluent conditions), and acetylated IRF-2 binds to the $\mathrm{H} 4$ promoter, thus participating in gene regulation for controlling cell growth. Acetylated IRF-2 may also interact with other proteins, resulting in more efficient gene transcription. Nucleolin, a nuclear protein, preferentially associates with acetylated IRF-2, over nonacetylated IRF-2, resulting in a more efficient transcription of the $\mathrm{H} 4$ gene [20] (Figure 1(b)).

IRF-2 binds to PCAF (and p300) and is then subject to acetylation at the lysine residue. Two lysine residues in the DBD, Lys-75, and Lys-78 are found to be the major acetylation sites of IRF-2. Lys-75 is the major site of acetylation by p300 and PCAF whereas Lys-78 is acetylated to a lesser degree. Lys-78 in IRF-2 is a residue conserved throughout members of the IRF family. Amino acid mutation of Lys78 in IRF-2 led to the abrogation of DNA binding activity independently of acetylation [14]. According to the crystal structure of the IRF-1 DNA-binding domain bound to a DNA target sequence, lysine 78 (corresponding to lysine 92 in IRF-7) stabilizes the protein-DNA complex through hydrogen bonding. Neutralization of the positive charge of this lysine residue effectively tightens the bonds between the transcriptional complex and the promoter [21]. Acetylation of lysine residue of IRF-2 does not alter its DNA binding 


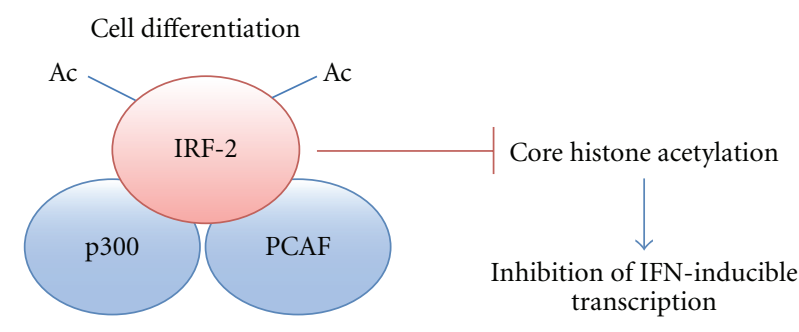

(a)

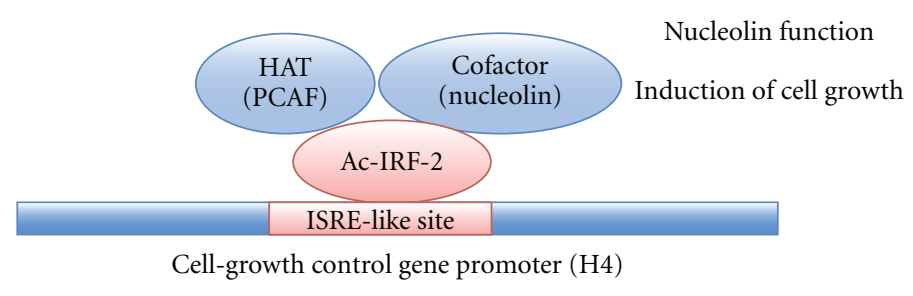

(b)

Figure 1: Two different functions of interferon regulatory factor-2 (IRF-2) through its acetylation by HAT. (a) During TPA-induced cell differentiation, IRF-2 binds histone acetylases, p300, and PCAF and inhibits core histone acetylation, resulting in the inhibition of IFN-inducible genes. (b) IRF-2 binds to PCAF and is acetylated. Acetylated IRF-2 is recruited to nuclear protein, nucleolin. The IRF2/PCAF/nucleolin complex contributes to cell growth through the activation of ISRE-like site in the H4 gene promoter.

ability. In the case of IRF-2, acetylation recruits cofactor (nucleolin) to the transcription factor-DNA complex [20].

As mentioned above, IRF-1 is a positive regulator for the IFN-producing system, and stimulates IFN- $\beta$ promoter with PCAF in the in vitro luciferase assay in cultured cells [13]. Dornan et al. reported that the interaction with IRF1 and p300 is important for synergizing activity of the p53-induced p21 gene activation, independent of IRF-1's DNA binding activity [22]. In their investigation, deletion of the p300-binding sites in IRF-1 eliminates the ability of IRF-1 to stimulate p53 acetylation and associated p53 activity. The nonacetylatable p53 mutant (p53-6KR) cannot be stimulated by IRF-1. They concluded that IRF-1 binding to $\mathrm{p} 300$ stabilizes $\mathrm{p} 300$ binding to the transactivation domain of p53, including p53 acetylation. Another report indicates that IRF-1 is acetylated and associates with HATs to form a complex that assembles on the HIV-1LTR promoter, and CBP is recruited by IRF-1 to HIV-1LTR promoter even in the absence of Tat [23].

\section{IRF-3 and IRF-7}

IRF-3 and IRF-7 are involved in IFN gene expression during viral infections. IRF-3 specifically targets IFN- $\alpha$ and IFN$\beta$ whereas IRF-7 is required for the induction of additional members of the IFN- $\alpha$ multigene family. In its inactive form, IRF-3 is restricted to the cytoplasm in unstimulated cells. Viral infection or treatment with dsRNA triggers a signal, which results in the specific phosphorylation of specific serine residues in IRF-3. The phosphorylated IRF-3 assembles into a homodimer and then forms a complex with the coactivators $\mathrm{CBP} / \mathrm{p} 300$ in the nucleus $[24,25]$. Specific phosphorylation sites such as Ser-386, were identified for IRF-3 dimerization (activation) [26, 27]. The histone acetyltransferase p300 is required for the DNA binding by the IRF- 3 holocomplex, which leads to IFN- $\beta$ transcriptional activation.

IRF-7 is activated by virus-induced phosphorylation on serine residues within the $\mathrm{C}$-terminal regulatory domain, resulting in induction of the IFN- $\alpha$ gene [28]. Caillaud et al. found that IRF-7 is acetylated by PCAF and GCN5 at a single lysine residue (Lys-92) which is located in the DBD and is conserved throughout the entire IRF family [7]. They reported that acetylated IRF-7 displays an impaired
DNA binding capability and that PCAF repression leads to decreased IRF-7 activity. One may conclude that acetylation of lys-92, negatively modulates IRF-7 DNA binding. As a general rule, if the acetylation occurs within a DNA-binding domain, it will repress the DNA binding, and if it occurs adjacent to a DNA-binding domain, then it will activate DNA binding. Not surprisingly, the precise effect of lysine acetylation depends on the location of the target residue. DNA binding ability is regulated by acetylation of target sites (amino acids) in the case of IRF-7 [7].

Yang et al. reported that IRF-3 and IRF-7 synergistically activate the virus-induced IFN- $\beta$ promoter in the presence of the $\mathrm{p} 300 / \mathrm{CBP}$ coactivator [29]. The IFN- $\beta$ promoter contains a binding site for the ATF-2/c-Jun, IRFs, and Rel families, and ATF-2, c-Jun, IRF-3, IRF-7, and NF-kB are associated with the IFN- $\beta$ promoter in virus-infected cells in vivo [30]. The effects of ATF-2/c-Jun, IRF-1, IRF-3/IRF-7, and NF-kB, on the transcription of -100IFNbCAT reporter in the presence or absence of mammalian p300/CBP have been studied. IRF-1 was able to activate -100IFNbCAT on its own, however, IRF-1 did not stimulate transcription when combined with other factors, more than the sum of their individual effects, regardless of whether or not p300/CBP was present. In contrast, IRF-3/IRF-7 with ATF-2/c-Jun, or NF-kB showed a synergistic effect in the presence of p300/CBP although only IRF-3/IRF-7 had no effect on the promoter [29]. Moreover, no synergy was observed in the absence of either p300/CBP or IRF-3/IRF-7. p300/CBP play an important role for the functional activation of both IRF-3 and IRF-7 upon virus infection.

\section{HATs in Interferon Signaling}

The transcription of the IFN- $\beta$ gene in response to a viral infection requires the assembly of an enhanceosome, consisting of the transcriptional activators NF-kB, IRF-1, ATF2/c-Jun and HMGI(Y) [31, 32]. The enhanceosome activates transcription by recruiting the $\mathrm{CBP} / \mathrm{p} 300$ coactivator that is associated with the Pol II holoenzyme complex. $\mathrm{CBP}$ and PCAF can acetylate $\mathrm{HMGI}(\mathrm{Y})$ at distinct lysine residues, causing opposing effects on IFN- $\beta$ gene expression. Acetylation of HMGI(Y) by CBP causes enhanceosome disruption leading to the termination of IFN- $\beta$ gene activation. 
Furthermore, CBP-enhanceosome interactions are responsible for the fast recruitment of the PolII holoenzyme, ensuring rapid activation of IFN- $\beta$ gene expression in response to viral infection [33]. In addition, viral infection induces recruitment of IRF- 2 to some of endogenous IFN- $\beta$ enhancers as part of the enhanceosome assembly process, and IRF2 incorporation into enhanceosomes restricts the number of IFN- $\beta$ promoters directing transcription [34]. The repression mechanism of IRF-2 against the enhanceosome has also been investigated. IRF-2 repressed transcription neither by competitive DNA binding nor by directly or indirectly inhibiting assembly of the basal transcriptional machinery directly or indirectly. Senger et al. demonstrated that incorporation of IRF-2 into IFN- $\beta$ enhanceosomes blocks recruitment of CBP and the associated RNA Pol II and strongly inhibits activation of transcription [34]. This inhibition is not mediated by another protein recruited by IRF-2, but rather by the IRF-2 repression domain itself, while the IRF-2 repression domain interacts with neither the activators nor CBP, but blocks recruitment of CBP.

In contrast, the requirement for deacetylase activity in IFN- $\alpha$-inducible gene regulation has been reported [35]. IFN- $\alpha$ stimulation induces local histone $\mathrm{H} 4$ deacetylation, and the deacetylase HDAC1 associates with both STAT1 and STAT2. The deacetylase activity of HDAC1 acts as a positive coactivator for ISGF3-dependent transcriptional responses and enhances IFN- $\alpha$-induced transcription. The requirement for deacetylase activity is shared by IFN- $\gamma$ signaling through STAT1. The role of HDAC1 in IFN responses may reflect an independent activity of HDACcontaining complexes that are associated with transcriptional repression [35].

\section{HDAC and IRF}

Histone deacetylases (HDACs) are also associated with IRFdependent transcription. For instance, the HPV E7 protein binds to HDAC and interferes with the transactivation function of IRF-1 by recruiting HDAC to the IFN- $\beta$ promoter [36]. HPV E7 interferes with the IFN-signaling function of IRF-1 by recruiting HDAC to the specific promoter. IRF-5, another IRF family member, is known to regulate proinflammatory cytokine expression [37]. Feng et al. showed that both HDACs and HATs associate with IRF5 and alter in its transcriptional activity [38]. They also demonstrated by using trichostatin A(TSA) that ISRE, IFNA, and IL- 6 promoters, but not TNF- $\alpha$, require HDAC activity for transactivation. The HATs CBP and p300 bind and acetylate IRF-5. HDAC and HATs are recruited to IRF-5 in a distinct region and play an important role for transcriptional induction of proinflammatory cytokines mediated by IRF-5.

\section{Another IRF Family, c-Myb, and Other Transcription Factors}

The IRF family shares homology with the myb oncoproteins. The $c-m y b$ proto-oncogene product (c-Myb) is a member of the helix-turn-helix transcriptional activator family. c-Myb regulates differentiation and proliferation in immature hematopoietic and lymphoid cells, although the relationship of the $\mathrm{c}-\mathrm{Myb}$ family to the interferon system remains undefined [39]. CBP binds via its KIX domain to the activation domain of c-Myb and mediates c-Myb-dependent transcriptional activation [40]. c-Myb interacts with CBP, and the acetylation of $\mathrm{c}-\mathrm{Myb}$ enhances both its affinity for CBP and its capacity for transactivation.

The current list of acetylated proteins includes, amongst others, p53, Myb, GATA-1, Sp1, and MyoD [2, 41, 42]. In most cases, this modification potentiates transcription. Acetylation causes enhanced sequence-specific DNA binding for transcription factors like p53, E2F, EFLF, p50, and PC4 whereas it reduces DNA binding for other factors like Foxo1, HMG1(Y), and p65. The impact upon DNA binding depends on the sites of acetylation. p53 is a sequence-specific transcription factor that is acetylated by p300/CBP [43], and acetylation at lysine residues in the C-terminus of p53 activates its DNA binding. Its function directly depends upon its ability to bind to the promoters of its regulatory genes in a sequence-specific fashion and thus maintain cellular homeostasis. NF-kB is involved in IFN signaling. The NF-kB/REL family of transcription factors pivotally controls the inflammatory and immune responses, as well as other genetic programs that are central to cell growth and survival. NF-kB acetylated by p300/CBP inhibits its interaction with $\mathrm{IkB}$ and induces translocation of the factor to the nucleus. Acetylation of NF-kB is regulated by the prior phosphorylation. HDAC3 deacetylates NF-kB, and enables it to bind IKB and causes its translocation into the cytoplasm. Thus, acetylation, like phosphorylation, is important for regulating the nuclear functions of NF-kB [44].

\section{Future Direction}

There are many reports about the hematopoietic and immune regulation of IRFs, which have been demonstrated using primary cells from mice [45-48]. However, it is unclear how acetylation or HAT association with IRFs function affect the immune and hematopoietic systems in vivo. In an IFN-regulating or cell growth control system in vitro, HATs are important factors for the correct functioning of IRFs as well as NF-KB and p53. IRFs/HATs complex may associate or correlate with other factors including NF-kB and p53. Recently, other posttranscriptional modification such as methylation, ubiquitination, and sumoylation have also been investigated for many transcription factors. Some of these modifications' targets are also lysine residues. A recent report showed that sumoylation of IRF-1 or IRF-2 regulates their transcriptional activities $[49,50]$. We should investigate how these modifications might crosstalk with each other to regulate the transcription factor-mediated homeostasis. Further studies are necessary for clarifying the interaction with acetylation and other modifications of transcription factors.

\section{Acknowledgments}

This research was supported by the Japan Society for the Promotion of Science and the Ministry of Education, Science, Sports, and Culture of Japan. 


\section{References}

[1] K. Batta, C. Das, S. Gadad, J. Shandilya, and T. K. Kundu, "Reversible acetylation of non histone proteins: role in cellular function and disease," Sub-Cellular Biochemistry, vol. 41, pp. 193-212, 2007.

[2] D. E. Sterner and S. L. Berger, "Acetylation of histones and transcription-related factors," Microbiology and Molecular Biology Reviews, vol. 64, no. 2, pp. 435-459, 2000.

[3] G. Mizuguchi, A. Vassilev, T. Tsukiyama, Y. Nakatani, and C. Wu, "ATP-dependent nucleosome remodeling and histone hyperacetylation synergistically facilitate transcription of chromatin," Journal of Biological Chemistry, vol. 276, no. 18, pp. 14773-14783, 2001.

[4] T. Taniguchi, "Transcription factors IRF-1 and IRF-2: linking the immune responses arid tumor suppression," Journal of Cellular Physiology, vol. 173, no. 2, pp. 128-130, 1997.

[5] T. Taniguchi, K. Ogasawara, A. Takaoka, and N. Tanaka, "IRF family of transcription factors as regulators of host defense," Annual Review of Immunology, vol. 19, pp. 623-655, 2001.

[6] A. Masumi and K. Ozato, "Coactivator p300 acetylates the interferon regulatory factor-2 in U937 cells following phorbol ester treatment," Journal of Biological Chemistry, vol. 276, no. 24, pp. 20973-20980, 2001.

[7] A. Caillaud, A. Prakash, E. Smith et al., "Acetylation of interferon regulatory factor-7 by p300/CREB-binding protein (CBP)-associated factor (PCAF) impairs its DNA binding," Journal of Biological Chemistry, vol. 277, no. 51, pp. 4941749421, 2002.

[8] Y. Nakatani, "Histone acetylases—versatile players," Genes to Cells, vol. 6, no. 2, pp. 79-86, 2001.

[9] A. Battistini, "Interferon regulatory factors in hematopoietic cell differentiation and immune regulation," Journal of Interferon and Cytokine Research, vol. 29, no. 12, pp. 765-779, 2009.

[10] T. Fujita, J. Sakakibara, Y. Sudo, M. Miyamoto, Y. Kimura, and T. Taniguchi, "Evidence for a nuclear factor(s), IRF-1, mediating induction and silencing properties to human IFNbeta gene regulatory elements," EMBO Journal, vol. 7, no. 11, pp. 3397-3405, 1988.

[11] H. Harada, T. Fujita, M. Miyamoto et al., "Structurally similar but functionally distinct factors, IRF-1 and IRF-2, bind to the same regulatory elements of IFN and IFN-inducible genes," Cell, vol. 58, no. 4, pp. 729-739, 1989.

[12] H. Harada, M. Kitagawa, N. Tanaka et al., "Anti-oncogenic and oncogenic potentials of interferon regulatory factors-1 and -2," Science, vol. 259, no. 5097, pp. 971-974, 1993.

[13] A. Masumi, I. M. Wang, B. Lefebvre, X. J. Yang, Y. Nakatani, and K. Ozato, "The histone acetylase PCAF is a phorbol-esterinducible coactivator of the IRF family that confers enhanced interferon responsiveness," Molecular and Cellular Biology, vol. 19, no. 3, pp. 1810-1820, 1999.

[14] A. Masumi, Y. Yamakawa, H. Fukazawa, K. Ozato, and K. Komuro, "Interferon regulatory factor-2 regulates cell growth through its acetylation," Journal of Biological Chemistry, vol. 278, no. 28, pp. 25401-25407, 2003.

[15] S. R. Haynes, C. Dollard, F. Winston, S. Beck, J. Trowsdale, and I. B. Dawid, "The bromodomain: a conserved sequence found in human, Drosophila and yeast proteins," Nucleic Acids Research, vol. 20, no. 10, p. 2603, 1992.

[16] C. Dhalluin, J. Carlson, L. Zeng, C. He, A. Aggarwal, and M. Zhou, "Sructure and ligand of a histone aceyltransferase bromodomain," Nature, vol. 339, no. 6735, pp. 491-496, 1999.

[17] L. Burýšek, W. S. Yeow, B. Lubyová et al., "Functional analysis of human herpesvirus 8 -encoded viral interferon regulatory factor 1 and its association with cellular interferon regulatory factors and p300," Journal of Virology, vol. 73, no. 9, pp. 73347342, 1999.

[18] P. S. Vaughan, F. Aziz, A. J. van Wijnen et al., "Activation of a cell-cycle-regulated histone gene by the oncogenic transcription factor IRF-2," Nature, vol. 377, no. 6547, pp. 362-365, 1995.

[19] R. Xie, A. J. van Wijnen, C. van der Meijden, M. X. Luong, J. L. Stein, and G. S. Stein, "The cell cycle control element of histone $\mathrm{H} 4$ gene transcription is maximally responsive to interferon regulatory factor pairs IRF-1/IRF-3 and IRF-1/IRF7," Journal of Biological Chemistry, vol. 276, no. 21, pp. 1862418632, 2001.

[20] A. Masumi, H. Fukazawa, T. Shimazu et al., "Nucleolin is involved in interferon regulatory factor-2-dependent transcriptional activation," Oncogene, vol. 25, no. 37, pp. 5113$5124,2006$.

[21] C. R. Escalante, J. Yie, D. Thanos, and A. K. Aggarwal, "Structure of IRF-1 with bound DNA reveals determinants of interferon regulation," Nature, vol. 391, no. 6662, pp. 103-106, 1998.

[22] D. Dornan, M. Eckert, M. Wallace et al., "Interferon regulatory factor 1 binding to p300 stimulates DNA-dependent acetylation of p53," Molecular and Cellular Biology, vol. 24, no. 22, pp. 10083-10098, 2004.

[23] G. Marsili, A. L. Remoli, M. Sgarbanti, and A. Battistini, "Role of acetylases and deacetylase inhibitors in IRF-1-mediated HIV-1 long terminal repeat transcription," Annals of the New York Academy of Sciences, vol. 1030, pp. 636-643, 2004.

[24] W. Suhara, M. Yoneyama, I. Kitabayashi, and T. Fujita, "Direct involvement of CREB-binding protein/p300 in sequencespecific DNA binding of virus-activated interferon regulatory factor-3 holocomplex," Journal of Biological Chemistry, vol. 277, no. 25, pp. 22304-22313, 2002.

[25] M. Yoneyama, W. Suhara, Y. Fukuhara, M. Fukuda, E. Nishida, and T. Fujita, "Direct triggering of the type I interferon system by virus infection: activation of a transcription factor complex containing IRF-3 and CBP/p300," EMBO Journal, vol. 17, no. 4, pp. 1087-1095, 1998.

[26] M. Mori, M. Yoneyama, T. Ito, K. Takahashi, F. Inagaki, and T. Fujita, "Identification of Ser-386 of interferon regulatory factor 3 as critical target for inducible phosphorylation that determines activation," Journal of Biological Chemistry, vol. 279, no. 11, pp. 9698-9702, 2004.

[27] D. Panne, S. M. McWhirter, T. Maniatis, and S. C. Harrison, "Interferon regulatory factor 3 is regulated by a dual phosphorylation- dependent switch," Journal of Biological Chemistry, vol. 282, no. 31, pp. 22816-22822, 2007.

[28] W. C. Au, W. S. Yeow, and P. M. Pitha, "Analysis of functional domains of interferon regulatory factor 7 and its association with IRF-3," Virology, vol. 280, no. 2, pp. 273-282, 2001.

[29] H. Yang, G. Ma, C. H. Lin, M. Orr, and M. G. Wathelet, "Mechanism for transcriptional synergy between interferon regulatory factor (IRF)-3 and IRF-7 in activation of the interferon- $\beta$ gene promoter," European Journal of Biochemistry, vol. 271, no. 18, pp. 3693-3703, 2004.

[30] M. G. Wathelet, C. H. Lin, B. S. Parekh, L. V. Ronco, P. M. Howley, and T. Maniatis, "Virus infection induces the assembly of coordinately activated transcription factors on the IFN- $\beta$ enhancer in vivo," Molecular Cell, vol. 1 , no. 4 , pp. 507$518,1998$.

[31] M. Merika, A. J. Williams, G. Chen, T. Collins, and D. Thanos, "Recruitment of $\mathrm{CBP} / \mathrm{p} 300$ by the IFN $\beta$ enhanceosome is 
required for synergistic activation of transcription," Molecular Cell, vol. 1, no. 2, pp. 277-287, 1998.

[32] N. Munshi, M. Merika, J. Yie, K. Senger, G. Chen, and D. Thanos, "Acetylation of HMG I(Y) by CBP turns off IFN $\beta$ expression by disrupting the enhanceosome," Molecular Cell, vol. 2, no. 4, pp. 457-467, 1998.

[33] J. Yie, K. Senger, and D. Thanos, "Mechanism by which the IFN- $\beta$ enhanceosome activates transcription," Proceedings of the National Academy of Sciences of the United States of America, vol. 96, no. 23, pp. 13108-13113, 1999.

[34] K. Senger, M. Merika, T. Agalioti et al., "Gene repression by coactivator repulsion," Molecular Cell, vol. 6, no. 4, pp. 931937, 2000.

[35] I. Nusinzon and C. M. Horvath, "Interferon-stimulated transcription and innate antiviral immunity require deacetylase activity and histone deacetylase 1," Proceedings of the National Academy of Sciences of the United States of America, vol. 100, no. 25, pp. 14742-14747, 2003.

[36] J. S. Park, E. J. Kim, H. J. Kwon, E. S. Hwang, S. E. Namkoong, and S. J. Um, "Inactivation of interferon regulatory factor1 tumor suppressor protein by HPV E7 oncoprotein. Implication for the E7-mediated immune evasion mechanism in cervical carcinogenesis," Journal of Biological Chemistry, vol. 275, no. 10, pp. 6764-6769, 2000.

[37] A. Takaoka, H. Yanai, S. Kondo et al., "Integral role of IRF5 in the gene induction programme activated by Toll-like receptors," Nature, vol. 434, no. 7030, pp. 243-249, 2005.

[38] D. Feng, N. Sangster-Guity, R. Stone et al., "Differential requirement of histone acetylase and deacetylase activities for IRF5-mediated proinflammatory cytokine expression," Journal of Immunology, vol. 185, no. 10, pp. 6003-6012, 2010.

[39] T. J. Gonda, "The c-Myb oncoprotein," International Journal of Biochemistry and Cell Biology, vol. 30, no. 5, pp. 547-551, 1998.

[40] Y. Sano and S. Ishii, "Increased affinity of c-Myb for CREBbinding protein (CBP) after CBP-induced acetylation," Journal of Biological Chemistry, vol. 276, no. 5, pp. 3674-3682, 2001.

[41] X. J. Yang, "Lysine acetylation and the bromodomain: a new partnership for signaling," BioEssays, vol. 26, no. 10, pp. 10761087, 2004.

[42] J. Boyes, P. Byfield, Y. Nakatani, and V. Ogryzko, "Regulation of activity of the transcription factor GATA-1 by acetylation," Nature, vol. 396, no. 6711, pp. 594-598, 1998.

[43] W. Gu and R. G. Roeder, "Activation of p53 sequence-specific DNA binding by acetylation of the p53 C-terminal domain," Cell, vol. 90, no. 4, pp. 595-606, 1997.

[44] L. F. Chen and W. C. Greene, "Shaping the nuclear action of NF- $\kappa \mathrm{B}$," Nature Reviews Molecular Cell Biology, vol. 5, no. 5, pp. 392-401, 2004.

[45] T. Matsuyama, T. Kimura, M. Kitagawa et al., "Targeted disruption of IRF-1 or IRF-2 results in abnormal type I IFN gene induction and aberrant lymphocyte development," Cell, vol. 75, no. 1, pp. 83-97, 1993.

[46] S. Hida, K. Ogasawara, K. Sato et al., "CD8+ T cell-mediated skin disease in mice lacking IRF-2, the transcriptional attenuator of interferon- $\alpha / \beta$ signaling," Immunity, vol. 13, no. 5, pp. 643-655, 2000.

[47] T. Sato, N. Onai, H. Yoshihara, F. Arai, T. Suda, and T. Ohteki, "Interferon regulatory factor-2 protects quiescent hematopoietic stem cells from type i interferon-dependent exhaustion," Nature Medicine, vol. 15, no. 6, pp. 696-700, 2009.

[48] A. Masumi, I. Hamaguchi, M. Kuramitsu et al., "Interferon regulatory factor-2 induces megakaryopoiesis in mouse bone marrow hematopoietic cells," FEBS Letters, vol. 583, no. 21, pp. 3493-3500, 2009.

[49] K. J. Han, L. Jiang, and H. B. Shu, "Regulation of IRF2 transcriptional activity by its sumoylation," Biochemical and Biophysical Research Communications, vol. 372, no. 4, pp. 772778, 2008.

[50] S. M. Park, M. Chae, B. K. Kim et al., "SUMOylated IRF-1 shows oncogenic potential by mimicking IRF-2," Biochemical and Biophysical Research Communications, vol. 391, no. 1, pp. 926-930, 2010. 

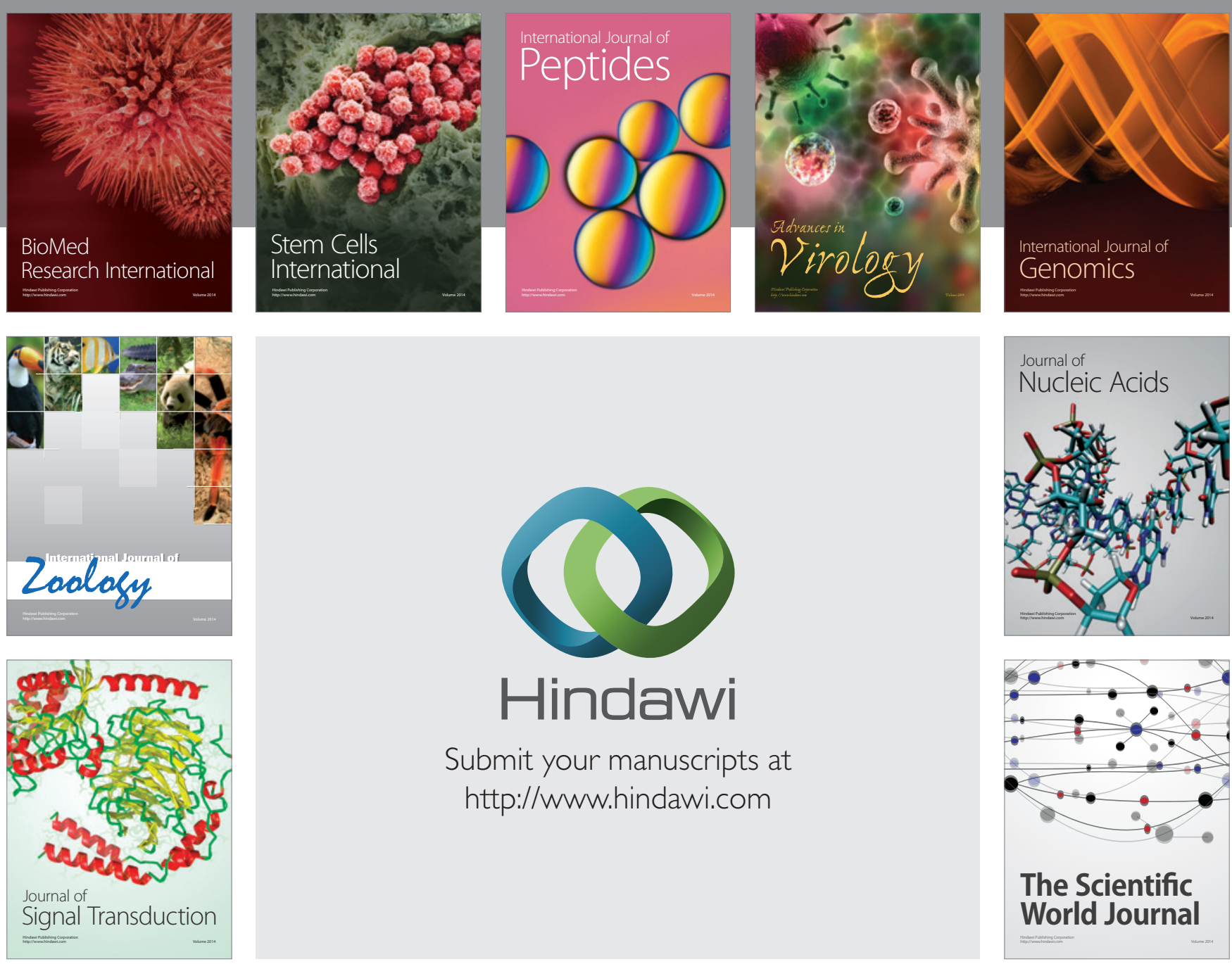

Submit your manuscripts at

http://www.hindawi.com
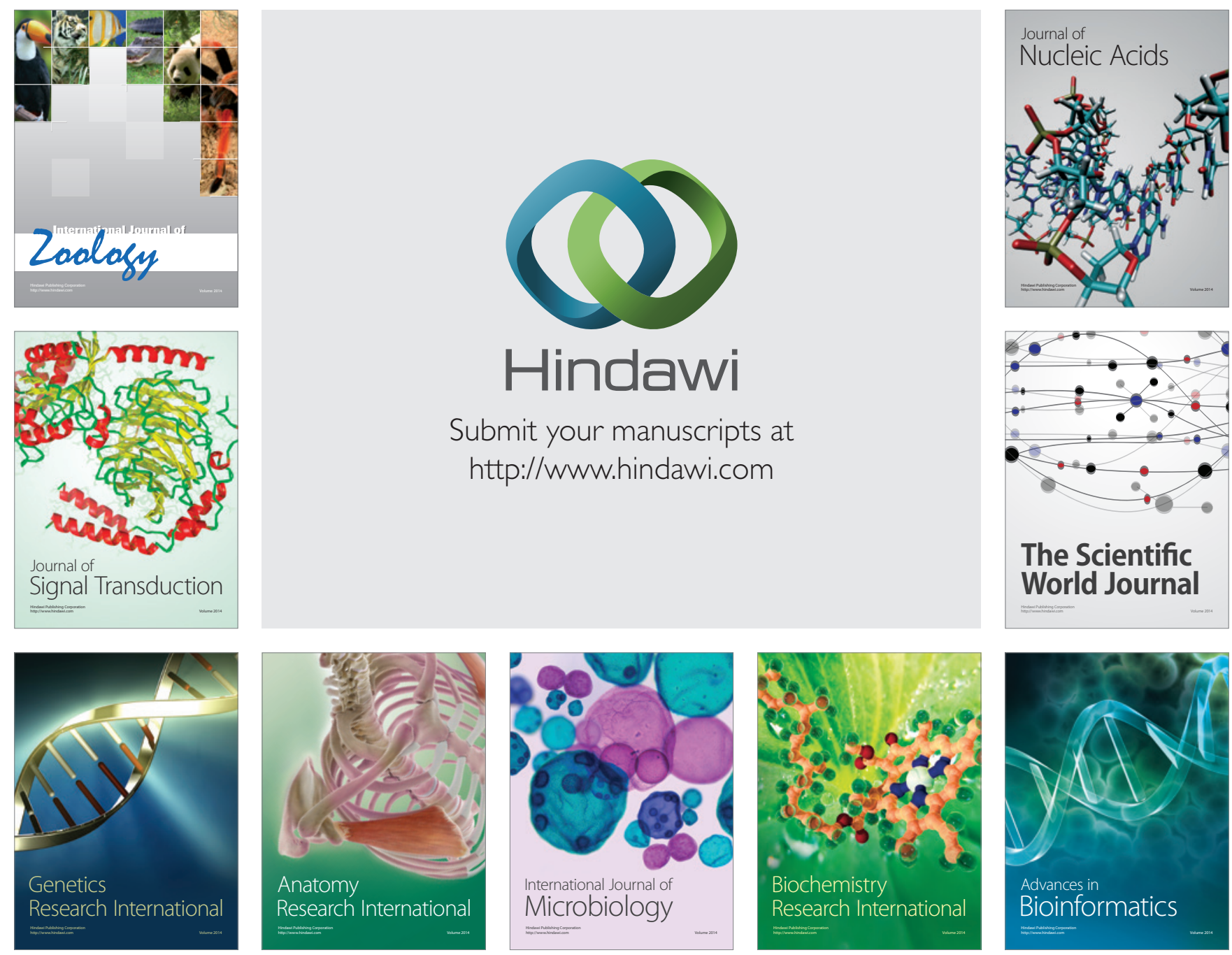

The Scientific World Journal
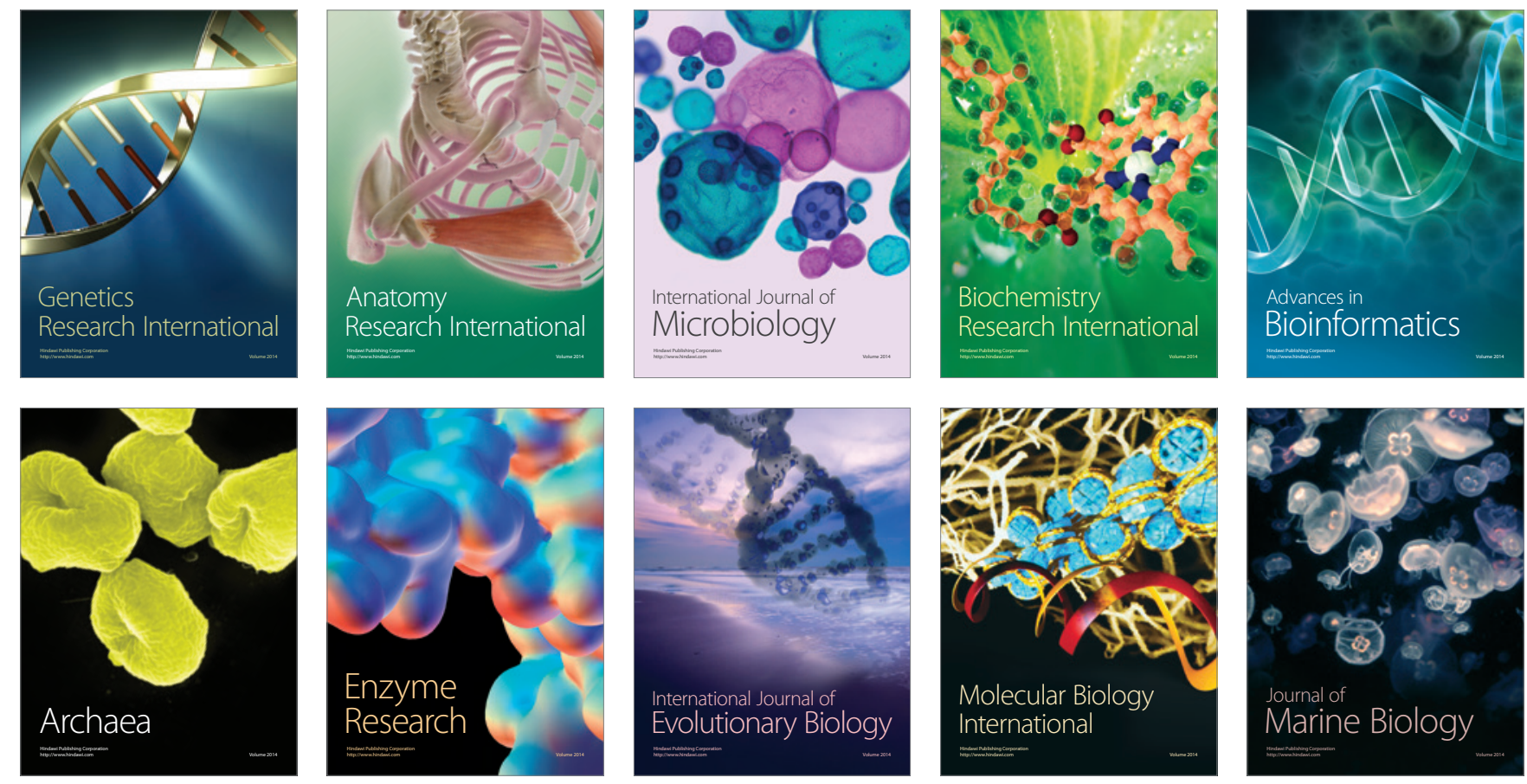